\title{
Logical Form through Abstraction
}

\author{
Zoltán Gendler Szabó \\ Yale University
}

DOI: $10.2478 /$ disp-2020-0012

BIBLID [0873-626X (2020) 58; pp. 251-263]

\begin{abstract}
In a recent book, Logical Form: between Logic and Natural Language, Andrea Iacona argues that semantic form and logical form are distinct. The semantic form of a sentence is something that (together with the meanings of its parts) determines what it means; the logical from of a sentence is something that (all by itself) determines whether it is a logical truth. Semantic form does not depend on context but logical form does: for example, whether 'This is this' is a logical truth depends on whether the two occurrences of 'this' are used to demonstrate the same individual. I respond by claiming that logical form is indifferent to reference and is sensitive only to obligatory co-reference. When the speaker intends both occurrences of 'this' to be interpreted the same way the logical from of 'This is this' is $a=a$, while in a context where the speaker has no such intention it is $a=b$. This proposal allows a much more conservative revision of the traditional picture than the one suggested by Iacona. Instead of identifying the logical form of a natural language sentence by seeking a formalization in an artificial language, we obtain it through abstraction from its syntactic analysis: replacing the non-logical expressions by schematic letters, making sure that we use identical ones if and only if the speaker intended co-reference.
\end{abstract}

\section{Keywords}

Abstraction, context-dependence, logic, logical form, semantics.

\section{Logically interpreted LF's}

"Logical form" is a technical term whose meaning and use has remained in flux despite various attempts to nail it down. ${ }^{1}$ Usually, the logical form of a sentence is supposed to do two things. Its semantic

\footnotetext{
${ }^{1}$ The term was originally introduced by Bertrand Russell in his 1914 Lowell lectures; cf. Russell 1993. For a survey of contemporary views on logical form, see Pietroski 2016.
} 
role is to determine, together with the meanings of the parts of the sentence, what the sentence means. Its logical role is to determine, together with the logical forms of other sentences within an inference, whether the inference is logically valid. That something can play both these roles is what Andrea Iacona, in his book Logical Form: Between Logic and Natural Language, calls the uniqueness thesis. ${ }^{2}$ The central claim of the book is that the uniqueness thesis is false: we should allow logical forms to do their work in logic and let something else pick up the slack in semantics.

What does 'determination' mean in the specification of the semantic and logical roles of logical form? Sometimes when we say that some facts determine others we mean that the latter hold solely in virtue of the former. For example, that fact that France is currently world champion in soccer is arguably determined in this sense by the fact that France defeated Croatia on July 15, 2018 in the FIFA World Cup Final in Moscow. I think the claim that logical from determines logical validity in this sense is false: no inference is logically valid solely in virtue of the fact that it has some form or other. Logical validity can only be explained by appeal to other, more general logical validities. ${ }^{3}$ The fact that (1) is an instance of (1a) goes some distance towards explaining its logical validity, the fact that it is an instance of (1b) goes further, and the fact that it is an instance of (1c) goes further still. But (1) is not logically valid solely because it has these forms, but rather, because it has these forms and because these forms themselves are logically valid.

(1) Venus is a star; therefore, Venus is a star or Venus is a hole in the firmament

(1a) $v$ is a star; therefore, $v$ is a star or $v$ is a hole in the firmament

(1b) $v$ is an $S$; therefore, $v$ is an $S$ or $v$ is an $H$

(1c) $p$; therefore, $p$ or $q$

What we need, I suggest, is a weaker explanatory notion of deter-

${ }^{2}$ Iacona 2018: 39.

${ }^{3}$ See Szabó 2012. 
mination: some facts determine some other facts if and only if the latter can be explained by (among other things) appeal to the former. Then, if the uniqueness thesis holds, the logical form of a sentence is a schema that abstracts from all and only those properties of the sentence that are irrelevant to its semantic or logical behavior.

What do logical forms in this sense look like? We can arrive at them thorough careful abstraction. Phonological properties of sentences can be abstracted away: when they make a difference to meaning (e.g. when the sentence contains an expression associated with focus, as 'only' is in 'John only introduced Bill to Sue' vs. 'John only introduced Bill to Sue' vs. 'John only introduced Bill to Sue') we can make sure that the relevant feature is represented in the syntax. ${ }^{4}$ Some but not all semantic properties can also be ignored. The logical behavior of 'Venus is a star' depends on the meanings of 'is' and 'a' but not on the meanings of 'Venus' or 'star' - uniformly substituting another proper count noun for the former or another common count noun for the latter within an inference won't affect its logical validity. ${ }^{5}$ When it comes to syntactic properties, logical forms can ignore almost nothing. Some syntactic theories posit uninterpreted features which are ultimately erased in the course of deriving the sentence - these certainly won't matter to logic. But it is hard to see how anything about the outcome of the derivation (what is sometimes called $\mathrm{LF}^{6}$ ) could be guaranteed to be logically otiose. As a first, very rough approximation, we might then say that the logical form of (2) is something like (2'):

(2) Venus is a star

${ }^{4}$ In the case of focus, this is normally done by adding a focus-feature to the appropriate constituent; cf. [John only introduced [Bill] to Sue] vs. [John only introduced Bill to $\left[\mathrm{Sue}_{\mathrm{f}}\right]$ vs. [John only [introduced Bill to Sue $]_{\mathrm{f}}$ ].

${ }^{5}$ Except, perhaps, in hyper-intensional contexts. Since I am inclined to think if $S_{1} \neq S_{2}$ 'Lucy believes that $S_{1}$ ' hardly ever logically entails 'Lucy believes that $S_{2}$ ', I also think that if we don't ignore hyper-intensional contexts the notion of logical form is trivialized.

${ }^{6}$ While the expression 'LF' was originally introduced as the abbreviation of 'logical form', nowadays the term is used in linguists for something that plays the semantic role traditionally associated with logical form. LF cannot play the logical role, since it leaves the interpretation of logical expressions open. 
(2') $\left[\operatorname{pres}\left[\left[{ }_{-\mathrm{N}(\text { proper, count })}\right]\left[b e\left[a[-\mathrm{N}(\text { common, count })]_{\mathrm{NP}}\right]_{\mathrm{VP}}\right]_{\mathrm{vP}}\right]_{\mathrm{TP}}\right]$

(The italics marks that the phonological features of an expression have been stripped away. 'pres' represents a present tense marker, which is moved to the front of the tense phrase leaving the tenseless root of 'is' behind.) Call this the logically interpreted LF of (2) - the outcome of the syntactic derivation of the sentence together with the interpretations of its logical morphemes (in this case, the present tense marker, the copular verb, and the indefinite article).

If an inference is logically valid, that can always be explained by (among other things) appeal to its logically interpreted LF. We know this, since we arrive at logically interpreted LF's through a process of abstraction that ignores only logically irrelevant properties. Logically interpreted LF's can also play the semantic role: given the logically interpreted LF of a sentence, and given the meanings of its non-logical expressions, a compositional semantics can explain why the sentence means just what it does. We know this because the process of abstraction that generates logically interpreted LF's does not ignore any aspect of the syntactic structure of the sentence. And so, the uniqueness thesis - understood as a weak explanatory claim-appears to be in a good shape. Why does Iacona think the thesis is false?

\section{Context and logical form}

Iacona's argument against the uniqueness thesis is straightforward: whatever plays the logical role must depend on context and whatever plays the semantic role cannot. Iacona makes a solid case for the first claim. Consider, for example, (3):

(3) That is that.

When uttered in a context where the speaker conspicuously demonstrates the same thing twice, the sentence expresses a logical truth. When uttered in a context where the speaker conspicuously demonstrates different things, the sentences expresses a falsehood. To play its logical role, the logical form of (3) must thus be sensitive to context.

The defense of the second claim is less persuasive. The natural 
suggestion would be to say that the logical form of (3) must be a schema that carries indices in the positions where the sentence contains the demonstrative pronoun 'that'. But this, Iacona argues, won't work: if one attempts "to provide a general treatment of contextsensitivity in terms of indexed structures based on semantic considerations, one will end up holding a radical view according to which context sensitivity reduces to some nonstandard form of ambiguity, so that every difference of truth conditions due to context sensitivity can be described in terms of a difference in syntactic structure." This would be a mistake, Iacona suggests, because the meaning of a sentence is an intrinsic property of the sentence, compositional semantics seeks to explain why the sentence has the meaning it does, and explanations of intrinsic properties must rely solely on other intrinsic properties. ${ }^{8}$

All three of these claims are contentious. I am open-minded about the first (with qualifications), ${ }^{9}$ accept the second, ${ }^{10}$ but reject the third. My height is intrinsic to me, why I have my height is explained by my genes and my nutrition, and yet, my nutrition is not intrinsic to me. And so, I see no reason to deny that intrinsic sentence meanings could be explained by positing extrinsic logical forms.

While I think Iacona's argument fails, I agree with him that we should not individuate logical forms using the referents of contextsensitive expressions. Here is my argument. Whatever semantically distinguishes a pure demonstrative and proper name that refer to the same thing in a given context is, I presume, logically irrelevant. ${ }^{11}$ If we decided that pure demonstratives contribute their referents (and

${ }^{7}$ Iacona 2018: 50.

${ }^{8}$ Iacona 2018: 66-7.

${ }^{9}$ Not all linguistic meaning is intrinsic. Some expressions mean what they do in virtue of conventions that are external to them. Still, it remains plausible that duplicates of sentences whose lexical components have the same meaning themselves have the same meaning. That is, sentence meaning may well be intrinsic modulo lexical meaning; see section 2.1 in Szabó 2011.

${ }^{10}$ For a defense, see Szabó 2019.

${ }^{11}$ The inference "Venus is a planet; therefore, something is called "Venus" is just as invalid as the inference 'This is a planet; therefore, something has been demonstrated'. It is possible for Venus to be an unnamed and undemonstrated planet. 
nothing else) to logical forms, by uniformity, we should say the same thing about proper names. But then we would have to say that (4) is unambiguously logically valid — which is something we shouldn't say. It would amount to giving up the apriori status of logic.

(4) Hesperus is Hesperus; therefore, Hesperus is Venus.

This leaves us with a question. We cannot say that (3) has a single logical form since it is true in some contexts but false in others. We should not say that (3) has infinitely many logical forms corresponding to each pair of things that can be demonstrated by the occurrences of 'that' within the sentence. So, how many logical forms does (3) have?

\section{Obligatory co-reference}

The answer, I suggest, is two: one where the two occurrences of 'that' are co-indexed, and one where they are not.

$$
\begin{aligned}
& \text { (3a') }\left[\text { pres }\left[\left[-\mathrm{N}(\text { proper, count) }]_{1}\left[\text { be }[-\mathrm{N} \text { (proper, count) }]_{1 \mathrm{VP}}\right]_{\mathrm{vP}}\right]_{\mathrm{TP}}\right]\right. \\
& \left(3 b^{\prime}\right)\left[\text { pres }\left[[-\mathrm{N} \text { (proper, count) }]_{1}\left[\text { be }[-\mathrm{N} \text { (proper, count) }]_{2 \mathrm{VP}}\right]_{\mathrm{vP}}\right]_{\mathrm{TP}}\right]
\end{aligned}
$$

Sameness of indices represents obligatory co-reference. (3a') is a logical truth, (3b') is not- even if the two occurrences of the demonstrative happen to pick out the same thing. Logic doesn't care about reference, but it does care about obligatory co-reference.

What determines whether the two occurrences of 'that' are obligatorily co-referential? The very same thing that settles the interpretation of ambiguous words: the speaker's communicative intentions constrained by semantics. When a pure demonstrative is used the speaker must somehow indicate its referent. One way to do this is to indicate that the referent is whatever the referent of an earlier occurrence of an expression is. If the speaker does this, the two occurrences are co-indexed-provided co-reference is not ruled out semantically — as it is, for example, in (5):

(5) \#That is those.

Of course, if (3) occurs within a larger linguistic context, we need to consider cross-sentential co-indexing as well. For example, (6) has 
twelve possible logical forms:

(6) That is that. But that isn't that.

$\begin{array}{llll}1 & 1 & 1 & 1 \\ 1 & 1 & 1 & 2 \\ 1 & 1 & 2 & 1 \\ 1 & 2 & 1 & 1 \\ 1 & 1 & 2 & 2 \\ 1 & 2 & 1 & 2 \\ 1 & \mathbf{2} & \mathbf{2} & \mathbf{1} \\ 1 & 2 & 2 & 2 \\ 1 & 1 & 2 & 3 \\ 1 & 2 & 1 & 3 \\ 1 & 2 & 3 & 1 \\ 1 & 2 & 3 & 4\end{array}$

But that does not mean that (3), as it occurs within (6), has more than two possible logical forms - it still the case that it has just two - one where the two occurrences of 'that' within it co-refer, and one where they do not.

Indices are not perfect for representing obligatory co-reference. In counting the logical forms for (6), we did not distinguish logical forms that can be obtained from one another by uniform substitution of indices. A more perspicuous representation would use brackets to indicate obligatory co-reference. The seventh logically interpreted LF of (6) - marked in boldface above - could, for example, be represented as follows: $:^{12}$

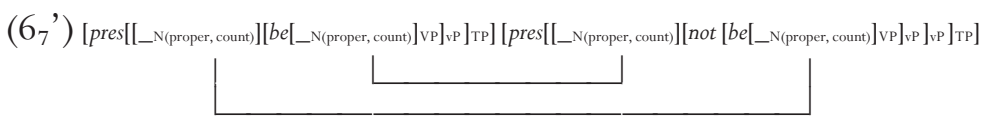

But since indices are standard fare in semantics and since brackets are typographically cumbersome, I will stick with the inferior notation and continue to talk of co-indexing.

${ }^{12}$ For the sake of simplicity, I have ignored 'but.' This discourse particle indicates contrast between the two sentences of (6). Discourse relations are often assumed to be logically irrelevant; whether this assumption is correct is controversial and I do not wish to take a stand on the matter here. 
Following the principle that we should represent pure demonstratives and proper names at the level of logical form the same way, I suggest that proper names should also bear indices. This fact explains two phenomena Iacona discusses. The first is the observation that the following passage seems to present us with a logically valid argument: "If Aristotle spent twenty years in the Academy and studied extensively the Dialogues, he admired Plato. But we know that he spent twenty years in the Academy and studied extensively the Dialogues. Therefore, the Stagirite admired Plato." 13 Yet, if we follow the standard practice and represent 'Aristotle' and 'the Stagirite' at logical form using different individual constants, we cannot predict this. On the other hand, if we assume that the speaker presumes that it is common ground that Aristotle is identical to the Stagirite we can allow co-indexing and derive a logically valid interpretation for the passage.

The second example is Kripke's Paderewski case. Peter, you might recall, fails to recognize that Paderewski the pianist is none other than Paderewski the politician. Since Peter would sincerely assent (in different contexts) both to 'Paderewski is a politician' and 'Paderews$\mathrm{ki}$ is not a politician' it appears that we must ascribe logical confusion to him despite his impeccable rationality. Indices, again, save the day. Given his beliefs, Peter clearly does not intend these two occurrences of 'Paderewski' to co-refer, so their logical forms will contain different indices. It is impossible for both of Peter's beliefs about Paderewski to be true, but they can be logically consistent nonetheless.

The fact that an occurrence of an expression obligatory co-refers with an occurrence of another is a logically significant fact. So, it must be represented at the level of logically interpreted LF's.

Whether we should say that indices are part of syntactic form is less clear. It is traditional to keep syntax restricted to sentences while - as (6) illustrates - obligatory co-reference can be crosssentential. There are also difficulties in integrating the indices proposed here with the ones posited by the Binding Theory. ${ }^{14}$ So, per-

${ }^{13}$ Iacona 2018: 74.

${ }^{14}$ According to Principle C of Chomsky 1981, a referring expression can never be co-indexed with anything. Fox 2000 argues that Principle $\mathrm{C}$ is operative at LF. Yet, the fact that definites (including pure demonstrative pronouns) can sometimes be bound - as illustrated, for example, by the sentence 'Mary talked 
haps we should say that co-indexing is a semantic matter. But since what is being indexed are occurrences of expressions, not expressions themselves, there is no easy way to add such an apparatus to traditional semantics. Being co-indexed is not a property of individual occurrences, but of collections. The best option is probably to relax the traditional formulation of the compositionality principle and allow that the meaning of a complex expression can depend not only on its syntactic form and the meanings of its constituents, but also on obligatory co-reference relations among occurrences of those constituents. ${ }^{15}$

Logical forms must display when occurrences of expressions obligatorily co-refer. This is no threat to the uniqueness thesis, but it does entail something surprising: since logical form depends on co-referential communicative intentions and since these intentions are not determined compositionally, logical from is not fully compositional. This vindicates the practice of always assigning logical forms to entire arguments, rather than to individual premises and conclusions.

\section{Comparison}

On Iacona's view logical forms are displayed by formulae that mirror their truth-conditions. The notion of truth-conditions is hyperintensional - 'Plato is a philosopher' and 'Either Plato is a philosopher or Plato is a philosopher and Aristotle is a philosopher' are said to have different truth-conditions. ${ }^{16}$ Whether a formula mirrors the truth-conditions of a sentence depends on whether it is an adequate formalization of the sentence. ${ }^{17}$ This view differs from the one advocated here in at least four ways.

to no senator without declaring afterwards that that was the one who would cosponsor her bill' from Elbourne (2008: 445) — is a problem for this view.

${ }^{15}$ See Fine 2007.

${ }^{16}$ Iacona 2018: 54.

${ }^{17}$ Iacona 2018: 59. 


\subsection{Stability}

What is adequate formalization? There is a clear interest-relative notion, according to which a formalization of an argument is adequate just in case it allows us to explain its logical properties. Given that notion, we should say that (7') is an adequate formalization of (7).

(7) Venus is a star; therefore, Venus is a star or Venus is a hole in the firmament

(7') $p \therefore p \vee q$

But (7') represents 'Venus is a star' simply with the schematic letter $p$, and this is obviously inadequate to explain the logical properties of many other inferences in which this sentence occurs. Iacona thinks adequate formalization must be more stable across linguistic contexts than what is dictated by the interest-relative notion of adequacy. But it is not clear where this extra stability is supposed to come from.

On my view, the source of stability is syntax. The LF of 'Venus is a star' distinguishes a number of constituents and these distinctions are preserved at the logically interpreted LF. There is a single logical form we can assign to (7), provided we assume that all occurrences of 'Venus' obligatorily co-refer and agree on which expressions within the inference should count as logical. This is the natural way to secure stability of logical form, but it is not available for those, like Iacona, who divorce logical and semantic form and tie syntax only to the latter. ${ }^{18}$

\subsection{Individuation}

Iacona stresses that his account leaves open the possibility that (8a) and (8b) are both adequately formalized as (8') and that (9a) and (9b) are both adequately formalized as $\left(9^{\prime}\right):^{19}$

\footnotetext{
${ }^{18}$ Note that on my view the logical from of a sentence never depends on the discourse in which it occurs. The logical form of a sentence depends on which of its expressions co-refer obligatorily, but it does not depend on which of its expressions co-refer obligatorily with expressions outside the sentence.

${ }^{19}$ Iacona 2018: 64-5.
} 
(8a) Not all Martians are green

(8b) Some Martians are not green

(8’) $\exists x(\operatorname{Martian}(x) \wedge \neg G r e e n(x))$

(9a) Donald is a drake

(9b) Donald is a male duck

(9') Male(d) $\wedge \operatorname{Duck}(d)$

In general, he thinks there is no clear rationale for ascribing logically equivalent sentences distinct logical forms, if the formulae have approximately the same complexity. Since I think logical forms have a semantic role to play I do have such a rationale: the logically interpreted LF of (8a) contains no expression that could be taken to be an existential quantifier and the logically interpreted LF of (9a) contains no expression that could be taken to be a predicate true of all and only ducks.

There is also independent reason to think that we should individuate logical forms more finely than Iacona suggests. Consider the minimal pair $(10 a, b)$ :

(10a) Some Martians are not green. They are dangerous.

(10b) Not all Martians are green. They are dangerous.

A natural reading of (10a) logically entails that some non-green Martians are dangerous. By contrast, (10b) has no such reading. Its only interpretation renders 'they' as a demonstrative referring to some contextually salient group of entities (perhaps all and only the Martians) — a reading (10a) has in addition to the one where 'they' refers to all and only the non-green Martians. To capture this logical difference we might, pace Iacona, want to assign distinct logical forms to the first sentences of (10a) and (10b) despite their truthconditional equivalence. ${ }^{20}$

${ }^{20}$ Since Iacona thinks logical forms are determined by truth-conditions, he can only accommodate this data by claiming that (10a) and (10b) have different truth-conditions. I think individuating truth-conditions finely is fine but I am 


\subsection{Formalism}

Iacona's logical forms are invariably displayed by formulae of artificial languages, while mine are displayed by symbolism designed for the study of natural languages. The formalism he employs is tailor-made for logic - accordingly, the (partial) explanations of logical validity he offers are more perspicuous than the ones I could give. That's fine: I never claimed that logically interpreted LF's are particularly useful in explaining logical validity. The claim is only that they can feature in such explanations.

\subsection{Logical constants}

The most important difference between Iacona's view and mine is that he thinks sentences have logical forms in virtue of their truthconditions, while I hold that sentences (or, rather, discourses) have logical forms in virtue of broadly structural features: syntax, indexing, and the meanings of logical constituents. On many views (Iacona himself is somewhat non-committal on this front), truthconditions are identified with propositions, and most ${ }^{21}$ propositions exist independently of how we represent the world. If we go along with this, Iacona's view entails that the question whether a particular inference is logically valid typically has an objectively correct answer (modulo indeterminacies about what propositions are expressed by the sentences within the inference). I reject this idea.

I think validity (necessary truth-preservation) is an objective matter, but I don't think there is an objective way to divide valid inferences into logical and non-logical ones. It is clear that 'or' and 'some' are logical expressions and that 'beech' and 'elm' are not. But almost all expressions semanticists are interested in are in the grey area inbetween. Should we say that 'true', 'must', 'before', 'do', the present tense, the imperative mood, the plural morpheme, the progressive marker, etc. are logical expressions? I doubt there is a principled

not sure individuating them this finely is a good idea. The difference in meaning between (8a) and (8b) has nothing to do with the conditions under which they are true and everything to do with the continuations they license.

${ }^{21}$ The ones that are about representations are exempted. 
response to these questions - what we end up doing depends on our explanatory purposes. On my view, the question whether some propositions logically entail others makes no sense, and the question whether some sentences logically entail others can only be answered relative to a choice of logical vocabulary.

Zoltán Gendler Szabó Yale University

\section{References}

Chomsky, N. 1981. Lectures on Government and Binding. Dordrecht: Foris. Elbourne, P. 2008. Demonstratives as individual concepts. Linguistics and Philosophy 31: 409-66.

Fine, K. 2007. Semantic Relationism. Oxford: Blackwell.

Fox, D. 2000. Economy and Semantic Interpretation. Cambridge, MA: MIT Press.

Iacona, A. 2018. Logical Form: Between Logic and Natural Language. Cham: Springer.

Pietroski, P. 2016. Logical form. In The Stanford Encyclopedia of Philosophy, ed. by E. N. Zalta. URL $=<$ https://plato.stanford.edu/archives/spr2016/entries/ $\operatorname{logical-form} />$.

Russell, B. 1993. Our Knowledge of the External World. New York: Routledge.

Szabó, Z. G. 2011. The case for compositionality. In The Oxford Handbook on Compositionality, ed. by W. Hinzen, E. Machery and M. Werning. Oxford: Oxford University Press.

Szabó, Z. G. 2012. Against logical form. In Donald Davidson on Truth, Meaning, and the Mental, ed. by G. Preyer. Oxford: Oxford University Press.

Szabó, Z. G. 2019. Semantic explanations. In Oxford Studies in the Philosophy of Language, ed. by E. Lepore and D. Sosa. Oxford: Oxford University Press. 